УДК 656.135:656.01

10.17213/2075-2067-2019-4-91-98

\title{
ОПЕРАТИВНОЕ УПРАВЛЕНИЕ ГРУЗОПОТОКАМИ В ТЕРМИНАЛЬНОЙ СИСТЕМЕ
}

\section{(C) 2019 г. Н. В. Напхоненко, М. Р. Караева}

\section{Южно-Российский государственный политехнический университет (НПИ), 2. Новочеркасск}

На основе анализа проблем оперативного управления транспортно-экспедиторской деятельностью терминала выявлены основные направления повышения эффективности технологических прочессов переработки и отправления грузов с применением логистических и экономико-математических методов. Выполнено построение модели оперативного управления работой терминала и обоснован критерий оптимальности при выполнении работ по переработке и отправлению грузов в междугородном сообщении.

Ключевые слова: транспортно-экспедиторская услуга; оптимизации оперативного управления транспортно-экспедиторской деятельностью терминала; логистические методы; издержки по переработке и отправлению грузов в междугородном сообщении; критерий оптимизации.

On the basis of the terminal transport-forwarding activity operational management problems analysis the main directions of increase in efficiency of technological processes of processing and freights departure with application of logistic and economic-mathematical methods are revealed. Creation of model of operational management of operation of the terminal is executed and the optimality criterion when working on processing and departure of freights in the long-distance message is reasonable.

Key words: transport-forwarding service; optimization of operational management of transport-forwarding activity of the terminal; logistic methods; costs for processing and departure of freights in the long-distance message; criterion of optimization.

Требования к качеству перевозок грузов в условиях их постоянно возрастающих объемов ставят новые задачи по развитию и эксплуатации грузовых терминалов, изысканию и использованию новых, более эффективных форм и методов работы, в первую очередь, за счет улучшения качества и совершенствования системы оперативного управления $[1,2]$.

Основными задачами оперативного управления являются обеспечение ритмичной работы всех звеньев производства, выявление дополнительных резервов перерабатывающих, пропускных и провозных возможностей автотранспорта и технических средств терминала, правильное их использование для успешного выполнения плановых заданий по переработке и отправлению грузов в междугородном сообщении.

Основой оперативного управления является оперативное планирование. В нашем случае оно включает в себя не только организацию и контроль, но также учет и анализ производственной деятельности, позволяющий своевременно обнаружить «узкие места» и устранить причины их возникновения. Существующий порядок составления оперативного плана транспортно-экспедиционной деятельности терминала заключается в опре- 
делении объемов работ на предстоящие сутки, в который включаются объемы отправления грузов по прямому варианту и через склады терминала на основании заключенных договоров с клиентурой, информации о прибытии транзитных автомобилей, а также исходя из наличия грузов на складах и контейнерных площадках терминала. На основании исходных и нормативных данных определяется количество, тип и модели подвижного состава, необходимые для перевозки грузов; разрабатываются маршруты движения автомобилей и число ездок; составляются заявки на выделение подвижного состава, в которых указывается наименование и вес груза, перечень экспедиторских и иных услуг при доставке; оформляются товарно-транспортные документы по маршрутам движения.

Практика работы терминалов показывает, что наиболее характерными причинами возникающих отклонений при выполнении суточного плана перевозки грузов и отправления транспортных средств по терминалу являются недостаточное количество транспортных средств для выполнения перевозок, нехватка погрузочно-разгрузочных механизмов (ПРМ) и персонала, несоответствие предоставленного подвижного состава (по количеству и моделям) запланированному, отказ грузополучателей от приема грузов [3-6]. Отмеченные недостатки в значительной степени могут быть устранены за счет повышения качества оперативного управления работой терминала, которое в настоящее время не осуществляет согласование предстоящего объема переработки грузов с фронтом погрузочно-разгрузочных работ и определяется в основном опытом работников, занятых на этом участке работы. Однако, даже в несложных ситуациях одного только опыта недостаточно для организации оптимальной работы терминала и автомобилей на линии. Возрастающие объемы перевозок и повышение уровня их централизации усложняют оперативное управление и требуют его совершенствования на основе применения логистических и экономико-математических методов при решении задач планирования. Если опыт руководителей производства в процессе управления вскрывает в основном причинные связи тех или иных явлений, то логистические и экономико-математи- ческие методы позволяют найти функциональную зависимость причин и следствий в конкретных задачах и получить алгоритмы для наилучшей организации процесса переработки и отправления грузов в междугородном сообщении.

При исследовании выявлено, что основные задачи, которые могут быть решены в технологических процессах переработки и отправления грузов с применением логистических и экономико-математических методов, заключаются: в разработке оптимального оперативного плана погрузки (выгрузки), исходя из количества груза, предъявленного к перевозке с наилучшим использованием грузоподъемности автомобилей и соблюдением норм времени их простоя; в оптимальном распределении погрузочно-разгрузочных механизмов по постам погрузки-разгрузки таким образом, чтобы обеспечить их наибольшую производительность; в определении оптимального варианта специализации складов терминала по родам грузов, погрузки, выгрузки, назначениям и рассортировке мелких партий; в расчете перерабатывающей способности складов [7-10].

Особенность оптимизации оперативного плана транспортно-экспедиторской деятельности терминала заключается в выборе наилучшего варианта организации процесса переработки и отправления грузов в междугородном сообщении, при котором учитывается оснащенность и техническое состояние объектов терминала, параметры технологических процессов, грузопотоки и объемы отправления, временные характеристики выполняемых операций.

Задача охватывает весь процесс перевозок от прибытия грузов на терминал и до отправки их грузополучателям, разгрузки и поступления новых партий груза на терминал [11-14]. Система оперативного управления работой терминала, построенная на основе логистического подхода, направлена на выбор наиболее эффективных форм организации перевозочного процесса и выполнения сопутствующего ему комплекса транспортно-экспедиторских услуг (ТЭУ), обеспечение дальнейшего снижения транспортных издержек народного хозяйства.

Снижение издержек по переработке и отправлению грузов в междугородном сообще- 
нии является важным резервом повышения эффективности работы грузового терминала, поэтому минимум затрат на осуществление погрузочно-разгрузочных, складских работ и выполнение экспедиторских операций принят в качестве критерия оптимальности оптимизационной логистической модели задачи оперативного планирования работы терминала $[15,16]$. Кроме того, критерий учитывает качественные показатели функционирования терминала, к которым относится время простоя подвижного состава, ПРМ и персонала. Оптимальность целевой функции достигается при условии полного использования ограниченных материальных и трудовых ресурсов.

Таким образом, целью задачи оперативного управления является оптимизация деятельности грузового терминала, направленная на рациональное использование подвижного состава, производственно-технической базы и трудовых ресурсов для обеспечения запланированного объема переработки и отправления грузов при минимуме всех издержек [17-20].

Общая постановка и построение экономико-математической модели оперативного планирования работы терминала состоит в следующем.

Терминалы отправляют грузы со складов грузоотправителя до складов грузополучателя (прямой вариант), а также с собственных складов. Грузы $k$-го вида $(k=1 \ldots K)$ перевозятся мало-, средне- и крупнотоннажными автомобилями $j$-го типа $(j=1 \ldots J)$ грузоподъемностью $q_{j}$ по $m$ маршрутом перевозок $(m=1 \ldots M)$. Коэффициент использования пробега на маршруте $-\beta_{m}$. Перевозимый груз обеспечивает использование грузоподъемности подвижного состава в соответствии с коэффициентом $j_{k}$. Оперативный план работы терминала представляет собой суточный план, а именно $N$ - массу груза, которая может быть принята от поставщиков, отправлена грузополучателям или оставлена на временное хранение.

Для переработки грузов терминал оснащен ПРМ $s$-го типа в количестве $B_{s}$ $(s=1 \ldots S)$, а также складами $r(r=1 \ldots R)$. $P_{r}$ соответствует предельной массе хранения грузов на $r$-м складе. При этом $W_{s}$ - производительность $s$-го типа ПРМ при погрузке-разгрузке за сутки; $g_{1 . r k}, g_{2 . r k}-$ масса $k$-го груза, хранящаяся на $r$-ом складе терминала на начало и конец суток, соответственно $(k=1 \ldots K ; r=1 \ldots R)$.

Автомобили, необходимые для выполнения суточного плана отправления и переработки грузов, условно делятся на два потока: $A_{j}$ - автомобили $j$-го типа $(j=1 \ldots J)$, выполняют работу по завозу и вывозу груза. Входящий и выходящий потоки автомобилей, в свою очередь, разделяются следующим образом:

$x_{1, j k m s}$ - количество автомобилей $j$-го типа, прибывших с $k$-м грузом по $m$ маршруту перевозок и оставшихся неразгруженными к концу суток, ед. $(j=1 \ldots J ; k=1 \ldots K$; $m=1 \ldots M ; s=1 \ldots S)$;

$x_{2, j k m s}-$ количество автомобилей $j$-го типа, прибывших с $k$-м грузом по $m$ маршруту перевозок и разгруженных $s$ типом ПРМ на склад терминала, ед. $(j=1 \ldots J ; k=1 \ldots K$; $m=1 \ldots M ; s=1 \ldots S)$;

$y_{1 . j k m s}-$ количество автомобилей $j$-го типа, предназначенных для работы по $m$ маршруту перевозок, простаивающих в ожидании освобождения $s$-го типа ПРМ или получения $k$-го вида груза, ед. $(j=1 \ldots J ; k=1 \ldots K$; $m=1 \ldots M ; s=1 \ldots S)$;

$y_{2 . j k m s}$ - количество автомобилей $j$-го типа, загруженных $s$-м типом ПРМ, ед. $(j=1 \ldots J$; $k=1 \ldots K ; m=1 \ldots M ; s=1 \ldots S)$;

$y_{3 . j k m s}$ - количество автомобилей $j$-го типа, вывозящих $k$-й груз по $m$ маршруту перевозок по прямому варианту $(j=1 \ldots J ; k=1 \ldots K$; $m=1 \ldots M)$.

За время в наряде $T_{\text {н }}$ автомобиль $j$-го типа простаивает в ожидании погрузки-разгрузки $s$-м типом ПРМ $t_{\mathrm{npj}}$ и осуществляет перевозки на $m$ маршруте со скоростью $V_{\text {тj}}$, при этом он выполняет $a_{j m}$ ездок.

Функциональные издержки терминала по переработке и отправлению грузов включают стоимость погрузочно-разгрузочных и складских работ и выполнения экспедиторских операций. Наряду с перечисленными затратами на практике имеют место потери, вызванные нарушениями нормального осуществления технологического процесса, которые связаны со сверхнормативными простоями транспортных средств и ПРМ по вине терминала.

Для определения суммарной величины затрат вводятся следующие обозначения. Затраты на: 
$C_{s}$ - погрузку-разгрузку 1 т груза $s$-м типом ПРМ, руб. $(s=1 \ldots S)$;

$C_{\text {скл }}$ - складскую переработку 1 т груза на складах терминала в течение суток, руб.;

$C_{\text {экп }}$ - выполнение экспедиторских операций, руб.;

$C_{\text {прj }}-1$ ч простоя автомобиля $j$-го типа, руб. $(j=1 \ldots J)$;

$C_{\text {прs }}-1$ ч простоя ПРМ $s$-го типа, руб. $(s=1 \ldots S)$.

Затраты на переработку и отправление грузов терминала рассчитываются по следующим составляющим элементам.

1. Затраты на погрузку-разгрузку.

Включают расходы на топливо для механизмов с тепловыми двигателями, на электроэнергию для электродвигателей и освещение, смазочные и обтирочные материалы, текущий и средний ремонт механизмов, амортизационные отчисления, замену оснастки, а также заработную плату (с начислениями во внебюджетные фонды) бригад, обслуживающих ПРМ, и накладные расходы.

Сумма затрат на погрузку-разгрузку по терминалу за сутки:

$$
\begin{aligned}
& \sum_{j=1}^{J} \sum_{k=1}^{K} \sum_{n=1}^{N} \sum_{s=1}^{S} C_{s} q_{j} j_{k} a_{j m} x_{1 . j k m s}+ \\
& +\sum_{j=1}^{J} \sum_{k=1}^{K} \sum_{n=1}^{N} \sum_{s=1}^{S} C_{s} q_{j} j_{k} a_{j m} x_{2 . j k m s}+ \\
& +\sum_{j=1}^{J} \sum_{k=1}^{K} \sum_{n=1}^{N} \sum_{s=1}^{S} C_{s} q_{j} j_{k} a_{j m} y_{1 . j k m s}+ \\
& +\sum_{j=1}^{J} \sum_{k=1}^{K} \sum_{n=1}^{N} \sum_{s=1}^{S} C_{s} q_{j} j_{k} a_{j m} y_{2 . j k m s} .
\end{aligned}
$$

2. Затраты на складские работы.

Включают расходы на внутрискладские погрузочно-разгрузочные работы (ПРР), эксплуатацию складов, а также заработную плату персонала, непосредственно связанного со складским хранением и переработкой грузов, с начислениями.

Сумма затрат на складские операции по терминалу за сутки:

$$
\begin{gathered}
\left(\sum_{r=1}^{R} \sum_{k=1}^{K} g_{1 . r k}+\sum_{j=1}^{J} \sum_{k=1}^{K} \sum_{n=1}^{N} \sum_{s=1}^{S} q_{j} j_{k} a_{j m} x_{2 . j k m s}-\right. \\
\left.-\sum_{j=1}^{J} \sum_{k=1}^{K} \sum_{n=1}^{N} \sum_{s=1}^{S} q_{j} j_{k} a_{j m} y_{2 . j k m s}\right) \cdot C_{\text {скл }} \cdot
\end{gathered}
$$

3. Затраты по выполнению экспедиторских операций.

Включают все расходы на содержание диспетчерских пунктов, амортизационные отчисления и текущий ремонт зданий и сооружений, заработную плату диспетчеров и других работников транспортной экспедиции с начислениями, прочие расходы.

Сумма затрат по терминалу:

$$
\begin{aligned}
& \left\{\sum _ { j = 1 } ^ { J } \sum _ { k = 1 } ^ { K } \sum _ { n = 1 } ^ { N } \sum _ { s = 1 } ^ { S } \left[q _ { j } j _ { k } a _ { j m } \left(x_{1 . j k m s}+x_{2 . j k m s} y_{1 . j k m s}+\right.\right.\right. \\
& \left.\left.\left.+y_{2 . j k m s}\right)\right]+\sum_{j=1}^{J} \sum_{k=1}^{K} \sum_{n=1}^{N} \sum_{s=1}^{S}\left[q_{j} j_{k} a_{j m} y_{3 . j k m s}\right]\right\} C_{\text {эксп }} .
\end{aligned}
$$

4. Стоимость потерь от простоя автомобилей на терминале за сутки:

$$
\sum_{j=1}^{J} \sum_{k=1}^{K} \sum_{n=1}^{N} \sum_{s=1}^{S}\left(x_{1 . j k m s}+y_{1 . j k m s}\right) \cdot C_{\operatorname{пр} j} t_{\text {пр } j} \cdot
$$

5. Величина потерь от простоя ПРМ:

$$
\sum_{s=1}^{S} Z_{s} \cdot t_{\text {пр } s} \cdot C_{\text {пр }},
$$

где $Z_{s}$ - количество простаивающих ПРМ.

Задача решается при следующих ограничениях.

1. Количество автомобилей $j$-го типа с $k$-м грузом, которые необходимо разгрузить на терминале, и автомобилей, оставшихся неразгруженными с предыдущих суток, не превышает общего количества автомобилей $j$-го типа, которым должны быть оказаны ТЭУ по ввозу за сутки:

$$
\sum_{k=1}^{K} \sum_{n=1}^{N} \sum_{s=1}^{S}\left(x_{1 . j k m s}+x_{2 . j k m s}\right) \leq A_{j},
$$

для $j=1 \ldots J ; k=1 \ldots K ; m=1 \ldots M ; s=1 \ldots S$.

2. Количество автомобилей $j$-го типа, вывозящих $k$-й груз по $m$ маршруту со складов грузоотправителей; автомобилей, которые должны быть загружены $s$-м типом ПРМ на складах терминала по плану предстоящих суток, и автомобилей, простаивающих в ожидании освобождения ПРМ или получения груза с предыдущих суток, не превышает общего количества автомобилей, которые необходимо загрузить по плану:

$$
\begin{gathered}
\sum_{k=1}^{K} \sum_{m=1}^{M}\left(y_{3 . j k m s}+\sum_{s=1}^{S}\left(y_{1 . k m s}+y_{2 . j k m s}\right) \leq H j\right. \\
\text { для } j=1 \ldots J ; k=1 \ldots K ; m=1 \ldots M ; s=1 \ldots S .
\end{gathered}
$$


3. Количество ПРМ s-го типа, используемых для погрузки-разгрузки, не превышает их общего количества на терминале:

$$
\begin{gathered}
\frac{1}{W_{s}} \cdot \sum_{j=1}^{J} \sum_{m=1}^{M} q_{j} j_{k} a_{j m}\left(x_{1 . j k m s}+x_{2 . j k m s}+y_{1 . j k m s}+\right. \\
\left.+y_{2 . j k m s}\right)+Z_{s}=B_{s},
\end{gathered}
$$

где $j=1 \ldots J ; m=1 \ldots M$.

4. Остаток грузов на складах терминала к концу планируемых суток равен сумме переходящего остатка грузов с предыдущих суток и поступивших грузов за вычетом массы грузов, отправленных со складок терминала:

$$
\begin{gathered}
\sum_{k=1}^{K} q_{2 r k}=\sum_{k=1}^{K} q_{1 r k}+ \\
+\sum_{k=1}^{K} j_{k} \sum_{j=1}^{J} q_{j} \sum_{m=1}^{M} a_{j m} \sum_{s=1}^{J}\left(x_{1 . j k m s}+x_{2 . j k m s}-y_{2 . j k m s}\right) \\
\text { для каждых } r=1 \ldots R, \\
\sum_{k=1}^{K} q_{2 r k}=\sum_{k=1}^{K} q_{1 r k}+ \\
+\sum_{k=1}^{K} j_{k} \sum_{j=1}^{J} q_{j} \sum_{m=1}^{M} a_{j m} \sum_{s=r+1}^{J}\left(x_{1 . j k m s}+\right. \\
\left.+x_{2 . j k m s}-y_{2 . j k m s}\right)
\end{gathered}
$$

для каждых $r=1 \ldots R ; j=1 \ldots J ; k=1 \ldots K$; $m=1 \ldots M ; s=1 \ldots S$.

Остаток грузов на складах терминала не отрицателен и не превышает емкости складов:

$$
0 \leq \sum_{k=1}^{K} g_{2 . r k} \leq \mathrm{P}_{r}
$$

для каждых $r=1 \ldots R ; k=1 \ldots K$.

5. Объем переработанных терминалом грузов (ввоз плюс вывоз) и складского хранения не превышают планового здания терминала на сутки:

$$
\begin{gathered}
\sum_{j=1}^{J} q_{j} a_{j m} \sum_{k=1}^{K} j_{k}\left[y_{3 . j k m s}+\right. \\
\left.+\sum_{s=1}^{S}\left(x_{1 . j k m s}+x_{2 . j k m s}+y_{2 . j k m s}\right)\right]=N,
\end{gathered}
$$

для каждых $j=1 \ldots J ; k=1 \ldots K ; m=1 \ldots M$; $s=1 \ldots S$.

6. При этом должно выполняться требование неотрицательности переменных:

$$
\begin{gathered}
x_{1 . j k m s} \geq 0 ; x_{2 . j k m s} \geq 0 ; \\
y_{1 . j k m s} \geq 0 ; y_{2 . j k m s} \geq 0 ; y_{3 . j k m s} \geq 0 .
\end{gathered}
$$

Вектор аргумент в сформулированной задаче $x_{j}$ - количество автомобилей, необходимое для выполнения программы перевозок $N$. Его можно определить по следующей формуле:

$$
\begin{gathered}
x_{j}=\sum_{j=1}^{J} \sum_{k=1}^{K} \sum_{m=1}^{M}\left[\sum _ { s = 1 } ^ { S } \left(x_{1 . j k m s}+x_{2 . j k m s}+\right.\right. \\
\left.\left.+y_{1 . j k m s}+y_{2 . j k m s}\right)+y_{3 . j k m s}\right]+\sum_{s=1}^{S} Z_{s},
\end{gathered}
$$

для каждых $j=1 \ldots J$.

Целевая функция модели имеет вид:

$$
\begin{aligned}
& F=\sum_{s=1}^{S}\left\{\sum _ { j = 1 } ^ { J } \sum _ { k = 1 } ^ { K } \sum _ { m = 1 } ^ { M } \left\{q _ { j } j _ { k } a _ { j m } \left[\left(C_{\text {пр } s}+\right.\right.\right.\right. \\
& \left.+C_{\text {эксп }}\right) x_{1 . j k m s}+\left(C_{\text {пр } s}+C_{\text {скл }}+\right. \\
& \left.+C_{\text {эксп }}\right) x_{2 . j k m s}+\left(C_{\text {пр } s}+C_{\text {эксп }}\right) y_{1 . j k m s}+ \\
& \left.+\left(C_{\text {пр } s}-C_{\text {скл }}+C_{\text {эксп }}\right)\right]+C_{\text {пр } j}\left(x_{2 . j k m s}+\right. \\
& \left.\left.\quad+y_{1 . j k m s}\right)\right\}+C_{\text {пр } s} Z_{s} \cdot t_{\text {пр } s}+ \\
& +\sum_{j=1}^{J} \sum_{k=1}^{K} \sum_{m=1}^{M}\left(q_{j} j_{k} a_{j m} y_{3 . j k m s}\right) C_{\text {эксп }}+ \\
& \quad+\sum_{k=1}^{K} \sum_{r=1}^{R} C_{\text {скл }} \cdot g_{1 . r k} \rightarrow \min .
\end{aligned}
$$

Расчет математической модели задачи выполняется по этапам.

Прежде всего, выделяется поток автомобилей, который подлежит загрузке в попутном направлении. Процент попутной загрузки составляет в среднем $18-20 \%$ от общего количества отправляемых автомобилей за сутки. Маршруты таких перевозок не постоянны и «непредсказуемы», поэтому возникает необходимость в ручном расчете при составлении оперативного плана. Машинная реализация задачи попутной загрузки привела бы к значительному усложнению модели, что не всегда оправдано. Попытка расширенной постановки задачи оперативного планирования неизбежно вызовет укрупнение исходной информации, потребует дополнительных допущений и ограничений, которые приведут к получению малонадежных результатов, поэтому предлагаемый способ расчета правомерен. Однако, несомненно, решение задачи загрузки автомобилей, следую- 
щих в попутном направлении, при выполнении МПГ представляет собой практический интерес и может служить предметом самостоятельного исследования.

Таким образом, расчет оперативного плана работы терминала заключается в определении необходимого количества ПРМ и автомобилей для отправки в междугородном сообщении по прямому варианту, в оптимизации работы терминала по переработке мелкопартионных и контейнерных грузов, следующих с перевалкой через склад.

Оптимальному варианту функционирования терминала соответствует минимальная сумма издержек от переработки и отправления грузов, включая издержки по отправлению автомобилей в попутном направлении, а также убытков от различного рода простоев транспортных средств и ПРМ. Разработанная экономико-математическая модель процесса оперативного управления применима для любых терминальных систем.

Применение разработанной логистической модели оперативного планирования работы терминала дает возможность регулирования уровня качества ТЭУ путем оптимизации работы подвижного состава и погрузочно-разгрузочных механизмов. Выбор и внедрение рациональной организации транспортно-экспедиторского процесса, направленной на снижение издержек по его осуществлению и повышение качества труда управленческого персонала, является одним из резервов повышения эффективности работы терминала.

\section{Литература}

1. Развитие терминальных систем [Электронный ресурс] — Режим доступа: https:// vuzlit.ru/1062299/razvitie_terminalnyh_sistem.

2. Постановление Правительства РФ от 20 декабря 2017 г. №1596 «Об утверждении государственной программы Российской Федерации «Развитие транспортной системы» (с изменениями и дополнениями). [Электронный ресурс] - Режим доступа: https://base.garant.ru/71843998/.

3. Миротин Л.Б. Интегральная логистика накопительно-распределительных комплексов (склады, транспортные узлы, терминалы). - М.: Экзамен, 2003. — 448 с.
4. Журавлев Н.П., Маликов О.Б. Транспортно-грузовые системы. - М.: Маршрут, 2006.

5. Маликов О.Б. Склады и грузовые терминалы. - М.: Издательский дом «БизнесПресса», 2005.

6. Гаджсинский А.М. Современный склад. Организация, технология, управление и логистика. - М.: ТК Велби, Изд-во Проспект, 2005.

7. Ensuring Competitiveness of Logistics Services by Selecting the Type of Storage SinglePiece Cargoes / И. М. Трунина, В.Г. Загорянский, Е. Л. Загорянская, Д.В. Малоштан: International Journal of Engineering \& Technology. — 2018. Vol. 7, Is. 4.3. - P. 537-540.

8. Напхоненко Н. В. Экономико-математическое моделирование управления грузопотоками в регионе в системе грузовых автостанций. // Научная мысль Кавказа. 2004. - Прил. Спецвып. №1. - С. 82-94.

9. Прокофьева Т.А., Лопаткин О.М. Логистика транспортно-распределительных систем: Региональный аспект. - М.: РКонсульт, 2003. $-400 \mathrm{c}$.

10. Логистика: интеграция и оптимизация логистических бизнес-процессов в целях поставок / В. В. Дыбская [и др.]. - М.: Эксмо, 2014. - 939 c.

11. Вельможин А.В., Гудков В.А., Миротин Л.Б. Теория организации и управления автомобильными перевозками: логистический аспект формирования перевозочных процессов. - Волгоград: РПК «Политехник», 2001. - $179 \mathrm{c}$.

12. Шехтер Д. Логистика. Искусство управления цепочками поставок / Д. Шехтер. - М.: Альпина, 2013. - 452 с.

13. Неруш Ю. М. Снабжение и транспорт: эффективное взаимодействие. - М.: Экономика, 1999.

14. Evers J., Loeve L., Lindeijer D. New Logistic Control: Concepts, Architecture and Logistics Programming. 3-th Intermational Meeting for Researth in Logistics. Trois-Rivieres, Canada. - 2000. - 15 p.

15. Кравченко Е.А., Бабий А.В., Уимаев E.Н. Эффективность терминальной системы перевозок грузов // Фундаментальные исследования. - 2007. - №12. - С. 61-65.

16. Бакаев А.А. Математические методы в планировании и экономических расчетах. - М.: Наука, 1987. - 223 с. 
17. Напхоненко Н. В., Загирняк Д. М., Караева М.P. Agricultural Cargo Transportation Logistics System Development // International Journal of Engineering \& Technology. 2018. — Vol. 7, Is. 4.3. - P. 185-190.

18. Напхоненко Н.В. Оптимизация логистических потоков в системе «грузоотправитель грузополучатель» для предприятий сельского хозяйства // Изв. вузов. Сев.-Кавк. регион. Техн. науки. - 2003. - Спецвыпуск. - С. 141-46.
19. Миротин Л. Б., Ташбаев Ы. Э., Порошина О.Г. Эффективная логистика. - М.: Экзамен, 2002. - 160 с.

20. Напхоненко Н.В. Экономико-математическое моделирование управления грузопотоками в регионе в системе грузовых автостанций. // Научная мысль Кавказа. 2004. - Прил. Спецвып. №1. - С. 82-94.

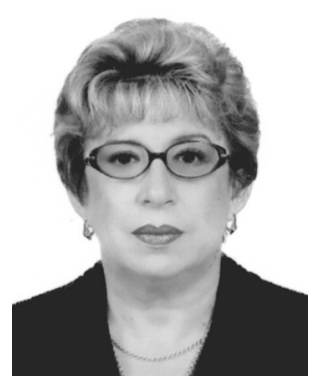

Напхоненко Наталья Васильевна - кандидат экономических наук, профессор кафедры «Производственный и инновационный менеджмент» ЮРГПУ (НПИ). Автор исследований по проблемам экономики и организации производства, экономике автомобильного транспорта, производственного менеджмента.

Napkhonenko Natalia Vasilievna - Ph.D., Candidate of Economics, professor of SRSPU (NPI) «Production and innovation management» department. Author of numerous research works, dedicated to problems of economics and production organization, economy of motor transportation, production management.

346428 , г. Новочеркасск, ул. Просвещения, 132

132 Prosveshcheniya st., 346428, Novocherkassk, Russia

Тел.: +7 (8635) 25-50-45; факс: +7 (8635) 22-72-69

E-mail: econ-en@mail.ru 


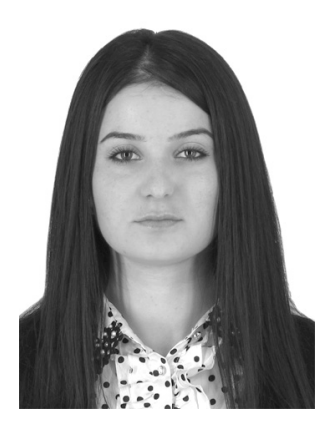

Караева Марина Руслановна - кандидат экономических наук, доцент кафедры «Международные логистические системы и комплексы» ЮРГПУ (НПИ). Автор работ по проблемам развития городского пассажирского транспорта.

Karayeva Marina Ruslanovna - candidate of economic Sciences, docent of «International logistics systems and complexes» Department of SRSPU (NPI). Author's works are devoted to problems of developing city's passenger transportation.

346428, г. Новочеркасск, ул. Троицкая, 126, кв. 111 126 Troitskaya st., app. 111, 346428, Novocherkassk, Russia Тел.: +7 (989) 613-66-62; e-mail: Karaevamarina@rambler.ru 